\title{
Alterações leucocitárias em pacientes com COVID-19 observadas em extensão de sangue periférico
}

\author{
Leukocytary changes in patients with COVID-19 observed in peripheral blood extension \\ Cambios leucocitarios en pacientes con COVID-19 observado en la extensión de sangre periférica
}

Recebido: 25/08/2021 | Revisado: 29/08/2021 | Aceito: 01/09/2021 | Publicado: 05/09/2021

\author{
Carolina Coradi \\ ORCID: https://orcid.org/0000-0001-7702-2505 \\ Universidade Paranaense, Brasil \\ E-mail: carolina_coradi@hotmail.com \\ Suellen Laís Vicentino Vieira \\ ORCID: https://orcid.org/0000-0002-7169-3671 \\ Universidade Paranaense, Brasil \\ E-mail: suellen.lais@hotmail.com
}

\begin{abstract}
Resumo
O objetivo do estudo foi verificar as principais alterações na linhagem leucocitária causadas pela infecção pelo SARS-CoV-2, vírus de origem zoonótica responsável pela doença conhecida como COVID-19. O SARS-CoV-2 afeta vários sistemas, incluindo o sistema hematopoiético, o que ocasiona alterações nas linhagens dos leucócitos. Os pacientes infectados pelo vírus SARS-CoV-2 apresentavam alterações numéricas e morfológicas na linhagem de leucócitos, sendo mais significativo a linfopenia e a neutrofilia. Quanto à morfologia, foram observadas várias anomalias. Os linfócitos reativos plasmocitóides e os semelhantes às células de Downey foram observados com frequência. Monócitos displásicos e neutrófilos com hipolobulação, classificados como anomalia Pseudo PelgerHuet adquirida, também estavam presentes. Essas anormalidades foram correlacionadas com maior chance de admissão na UTI e evolução para óbito. Houve correspondência entre a contagem normal de eosinófilos com a progressão positiva da doença, o que sugere que, a regeneração dos eosinófilos esteja relacionada com a recuperação da doença. Esse conjunto de alterações não foi observado em nenhuma outra pneumonia de origem viral. A contagem diferencial de leucócitos é um exame rápido e de fácil realização, que pode auxiliar a equipe médica na classificação de pacientes graves ou não graves, e fornecer direcionamento da evolução da doença.

Palavras-chave: Coronavírus; Leucócitos; Hematologia; Infecção por SARS-CoV-2; Contagem de leucócitos.
\end{abstract}

\begin{abstract}
The aim of the study was to verify the main alterations in the leukocyte lineage caused by infection with SARSCoV-2, a virus of zoonotic origin responsible for the disease known as COVID-19. SARS-CoV-2 affects several systems, including the hematopoietic system, which causes changes in leukocyte lineages. Patients infected with the SARS-CoV-2 virus had numerical and morphological alterations in the leukocyte lineage, with lymphopenia and neutrophilia being more significant. As for morphology, several anomalies were observed. Plasmacytoid and Downey cell-like reactive lymphocytes were frequently observed. Dysplastic monocytes and hypolobulated neutrophils, classified as acquired Pseudo Pelger-Huet anomaly, were also present. These abnormalities were correlated with a greater chance of admission to the ICU and progression to death. There was a correspondence between the normal eosinophil count and the positive progression of the disease, suggesting that eosinophil regeneration is related to disease recovery. This set of changes has not been observed in any other viral pneumonia. The differential leukocyte count is a quick and easy test to perform, which can help the medical team to classify patients with serious or non-serious conditions, and provide guidance on the evolution of the disease.
\end{abstract}

Keywords: Coronavirus; Leukocytes; Hematology; SARS-CoV-2 infection; WBC count.

\section{Resumen}

El objetivo del estudio fue verificar las principales alteraciones en el linaje leucocitario provocadas por la infección por SARS-CoV-2, virus de origen zoonótico responsable de la enfermedad conocida como COVID-19. El SARSCoV-2 afecta a varios sistemas, incluido el sistema hematopoyético, que provoca cambios en los linajes de los leucocitos. Los pacientes infectados por el virus SARS-CoV-2 presentaron alteraciones numéricas y morfológicas en el linaje leucocitario, siendo más significativas la linfopenia y la neutrofilia. En cuanto a la morfología, se observaron varias anomalías. Con frecuencia se observaron linfocitos plasmocitoides y linfocitos reactivos similares a células de Downey. También estaban presentes monocitos displásicos y neutrófilos hipolobulados, clasificados como anomalía adquirida de Pseudo Pelger-Huet. Estas anomalías se correlacionaron con una mayor probabilidad de ingreso en la UCI y progresión a la muerte. Hubo una correspondencia entre el recuento normal de eosinófilos y la 
progresión positiva de la enfermedad, lo que sugiere que la regeneración de eosinófilos está relacionada con la recuperación de la enfermedad. Este conjunto de cambios no se ha observado en ninguna otra neumonía viral. El recuento diferencial de leucocitos es una prueba rápida y fácil de realizar, que puede ayudar al equipo médico a clasificar a los pacientes con afecciones graves o no graves, y brindar orientación sobre la evolución de la enfermedad.

Palabras clave: Coronavirus; Leucocitos; Hematología; Infección por SARS-CoV-2; Recuento de leucocitos.

\section{Introdução}

No final do ano de 2019 a China se tornou o foco do mundo devido à identificação de um novo coronavírus, na cidade de Wuhan. Esse patógeno foi nomeado como SARS-CoV-2 e pertence há uma grande família de vírus que causam resfriados comuns e doenças respiratórias graves, como a Síndrome Respiratória do Oriente Médio (SROM - conhecida do inglês MERS - Middle East Respiratory Syndrome) e a Síndrome Respiratória Aguda Grave (SRAG - conhecida do inglês SARS - Severe Acute Respiratory Syndrome) (Tan L. et al., 2020).

O vírus SARS-CoV-2 é responsável por uma doença multissistêmica, possivelmente de origem zoonótica, chamada COVID-19. A COVID-19 possui um período de incubação de um a 14 dias. A infecção pode ser assintomática ou sintomática, sendo que os sintomas mais comuns são tosse, febre e dispnéia, podendo evoluir para SRAG e óbito (Shahri; Niazkar \& Rad, 2020).

Dentro de poucos meses a doença já havia se espalhado por todos os continentes e no dia 11 de Março de 2020 a Organização Mundial da Saúde (OMS) caracterizou a COVID-19 como pandemia devido a alta taxa de morbidade e mortalidade. Até o dia 11 de junho de 2021, mais de 174 milhões de casos de COVID-19 já foram registrados, incluindo mais de 3 milhões de mortes (OPAS, 2020).

O papel essencial dos elementos sanguíneos na cicatrização de tecidos já foi descrito há décadas. Os leucócitos, principalmente, desempenham papel fundamental na defesa do organismo contra agentes invasores, visto que, são células especializadas em combater agentes nocivos e manter a homeostasia do organismo frente a situações que comprometam a sua integridade (Falcão \& Calado, 2001).

Sabe-se que o SARS-CoV-2 afeta a hematopoiese e o sistema imunológico durante seu desenvolvimento (Ahnach et al.., 2020), isso ocasiona danos aos leucócitos e, por consequência, causa uma resposta imune ineficiente fazendo com que o organismo fique mais vulnerável a infecções (Blomme et al., 2020). Portanto, a observação dos parâmetros hematológicos concedidos pelo hemograma de rotina são de grande valia para acompanhar a progressão da doença (Sun S. et al., 2020).

\section{Metodologia}

O presente trabalho trata-se do levantamento dos principais achados na literatura sobre alterações leucocitárias em pacientes infectados com SARS-CoV-2. Utilizou os descritores: COVID-19, alterações hematológicas, SARS-CoV-2, hemograma na COVID-19. Considerou-se artigos científicos, livros, monografias, dissertações, teses e informações em sites oficiais. Os bancos de dados utilizados foram Medical Literature Analysis and Retrieval System Online (MEDLINE), Scientific Eletronic Library Online (Scielo), Google Acadêmico e Periódicos da Coordenação de Aperfeiçoamento de Pessoal de Nível Superior (CAPES) (Estrela, 2018).

\section{Resultados e Discussão}

\subsection{SARS-CoV-2}

Os coronavírus são vírus envelopados que pertencem à família Coronaviridae; subfamília Orthocoronavirinae; gênero Betacoronavírus; subgênero Sarbecovírus e espécie SARS-CoV-2. Vírus de genoma de RNA com fita simples (Khalil 
\& Khalil, 2020), o SARS-Cov-2 é altamente infeccioso e transmissível entre pessoas através de gotículas de saliva e secreções respiratórias (Mina, Van Besien \& Platanias, 2020).

O Betacoronavírus, quando observado por microscopia crioeletrônica, apresenta-se na forma de partículas esféricas e com diâmetro variável (cerca de $100 \mathrm{~nm}$ ). Possui viroplasma denso delimitado por uma bicamada lipídica da qual sobressaem as espículas da glicoproteína S (Ke et al., 2020).

SARS-CoV-2 contém quatro proteínas estruturais ( $\mathrm{S}, \mathrm{E}, \mathrm{M}$ e N) e dezesseis proteínas não estruturais (nsp1-16). O genoma é embalado por um envelope que está associado a três proteínas estruturais: proteína de membrana $(M)$, proteína de pico $(S)$ e proteína de envelope (E) (Wang et al., 2020) (Figura 1).

Figura 1. Estrutura típica do SARS-Cov-2 (80-120 nm), exibindo as proteínas estruturais E, N, M e S e seu genoma.

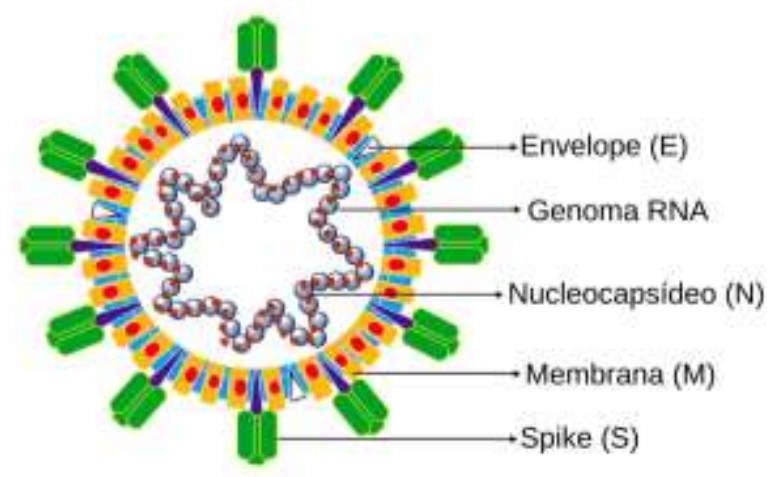

Fonte: Ke et al (2020).

A entrada do vírus nas células hospedeiras é mediada pela interação da glicoproteína de pico (S) e o receptor nas células hospedeiras. A glicoproteína S compreende duas subunidades funcionais: subunidade S1 e S2. A S1 é responsável por ligar-se ao receptor na célula hospedeira e a S2 pela fusão das membranas viral e celular (Walls et al., 2020).

\subsection{Fisiopatologia da COVID-19}

A porta de entrada para o vírus SARS-CoV-2 nas células hospedeiras é através da ligação da sua glicoproteína (S) ao receptor de enzima conversora de angiotensina 2 (ECA2) (Helal et al., 2020) que é expresso em diversos tecidos, como rim, miocárdio, sistema respiratório e hematopoiético (Choi et al., 2020). Ao ligar-se a ECA2 presente no epitélio alveolar, causa os sintomas respiratórios habitualmente observados nos pacientes, como tosse seca e dispnéia. Porém, o vírus também liga-se às células endoteliais, causando dano e iniciando um processo de agregação plaquetária, que pode resultar em trombose e/ou isquemia (Christensen et al., 2020). Nesse momento, há também a piora das lesões torácicas, aumento exorbitante de marcadores inflamatórios e pronunciada linfopenia. Esse aumento acelerado de citocinas circulantes está sendo chamado de "tempestade de citocinas" e é caracterizado pelo aumento exagerado de vários marcadores inflamatórios, que são capazes de esgotar o sistema imunológico, resultando em hematopoiese defeituosa e ativação da cascata de coagulação (Mina, Van Besien \& Platanias, 2020). A concentração maior de citocinas inflamatórias está relacionada com a gravidade da doença (Sun S. et al., 2020).

Embora os sintomas iniciais dos pacientes infectados pelo SARS-CoV-2 sejam respiratórios, a COVID-19 é uma doença multissistêmica, que afeta diversos órgãos, incluindo o sistema imunológico e hematopoiético (Lüke et al., 2020). A maioria dos pacientes apresenta alterações no sistema sanguíneo periférico (Sun S. et al., 2020), mas além das anormalidades hematológicas quantitativas, várias anomalias morfológicas foram descritas (Schapkaitz, Jager \& Levy, 2020) sugerindo que o SARS-CoV-2 produza efeitos diretos sobre as populações de células primitivas e maduras (Ropa et al., 2020). 
Ropa et al. (2020), sugerem que a proteína S recombinante possa promover alterações fenotípicas nas células hematopoiética primitivas e maduras, o que pode ser a explicação para a desordem das linhagens leucocitárias. Porém, é importante ressaltar que as células progenitoras hematopoiéticas não foram bem estudadas no contexto da COVID-19.

Em suma, acredita-se que a tempestade de citocinas aumenta o estresse da medula óssea, que resulta em produção e maturação defeituosa dos leucócitos. Portanto, as alterações hematológicas são, possivelmente, subprodutos da resposta imune antiviral do hospedeiro (Sadigh et al., 2020).

\subsection{Alterações hematológicas na infecção pelo SARS-CoV-2}

O hemograma é um dos exames de sangue mais regularmente solicitados por profissionais de saúde a seus pacientes. Esse exame demonstra principalmente os elementos sólidos do sangue (eritrócitos, leucócitos e plaquetas), desde características qualitativas e quantitativas das mesmas (Celkan, 2020). O hemograma é muito utilizado para auxiliar no diagnóstico de anemias, cânceres hematológicos, estados hemorrágicos, entre outras enfermidades.

O hemograma completo se divide em três secções: eritrograma, leucograma e plaquetograma. O eritrograma avalia quali-quantitativamente os eritrócitos e a hemoglobina presente no interior das hemácias. Com o leucograma é possível observar características de respostas imunológicas, devido a alterações apresentadas neste conjunto de células (neutrófilos, eosinófilos, basófilos, linfócitos e monócitos, além de células precursoras jovens destas). Já o plaquetograma, permite uma avaliação quali-quantitativa das plaquetas (Silva et al., 2016).

A extensão de sangue periférico, também chamado de esfregaço sanguíneo, além de permitir uma contagem relativa de leucócitos, é um teste rápido, barato e de fácil execução, que fornece resultados em um curto espaço de tempo, tornando-se muito acessível (Khartabil et al., 2020).

Assim como em diversas enfermidades, o hemograma apresenta alterações significativas em pacientes com COVID19 (Berber et al., 2020). Os parâmetros hematológicos dos pacientes vêm sendo cada vez mais abordados em diversos estudos por serem características importantes da doença. Os achados laboratoriais mais frequentes são leucocitose, linfopenia, neutrofilia e eosinopenia e sugere-se que estão diretamente relacionadas com o mau prognóstico da doença (Amgalan \& Othman, 2020).

As anormalidades hematológicas são observadas com mais frequência em pacientes com doença grave. Embora as complicações hemorrágicas possam estar presentes, devido ao desenvolvimento de Coagulação Intravascular Disseminada (CID), a maioria dos pacientes manifesta episódios trombóticos que podem ser venosos ou arteriais (Mina, Van Besien \& Platanias, 2020) e estão relacionados com maior fatalidade da doença (Liao et al., 2020).

\subsubsection{Linfócitos}

Os linfócitos são células sanguíneas com diferentes funções. Possuem tamanho de 6 a $15 \mu \mathrm{m}$, forma regular e arredondada, alta relação núcleo/citoplasma. Seu núcleo é de coloração azul-arroxeada e citoplasma escasso e basofílico e compreendem cerca de 20 a 50\% do total de leucócitos circulantes (Falcão \& Calado, 2001).

A linfopenia, redução quantitativa de linfócitos sanguíneos, é recorrente em infecções comuns e é o achado laboratorial mais frequente na COVID-19 (Asghar M. et al., 2020). Sabe-se que os pacientes com linfopenia grave têm maior risco de admissão em unidades de terapia intensiva e progressão para óbito durante a hospitalização (Shahri, Niazkar \& Rad, 2020), portanto, a linfopenia pode ser considerada um marcador para a gravidade da doença (Helal et al., 2020).

Embora mais estudos sejam necessários, várias teorias foram difundidas para tentar explicar o mecanismo para a linfopenia evidente observada na COVID-19 (Helal et al., 2020). Estes incluem que o receptor ECA2 é a porta de entrada para o SARS-CoV-2 e está expresso em diversos tecidos, incluindo os linfócitos, isso faz com que o vírus infecte diretamente essas 
células levando a lise e consequente linfopenia (Choi et al., 2020; Shahri, Niazkar \& Rad, 2020). Foi relatada a presença de linfócitos infiltrados no interstício pulmonar, o que pode ter relação com o aumento do consumo destes leucócitos (Sun S. et al., 2020). Além do mais, o excesso de quimiocinas e citocinas pró-inflamatórias circulantes observadas da infecção, desregula a via apoptótica dos linfócitos T, que também resulta em morte celular (Helal et al., 2020). Outra possível é o fato da administração de medicações esteroidais durante o curso da doença, que causam diminuição de linfócitos T circulantes (Shahri, Niazkar \& Rad, 2020).

Quanto à morfologia, foram identificados linfócitos reativos altamente pleomórficos (Gérard, Henry \& Thomas, 2020). Jamal et al. (2020), classificaram os linfócitos reativos em duas categorias, os com características altamente plasmocitóides, que são pequenos linfócitos maduros com núcleo excêntrico e citoplasma azul escuro; nesta categoria também estão às células com características blásticas, caracterizadas por células maiores com cromatina frouxa e nucléolo evidente. Na outra categoria foram enquadradas as células com morfologia semelhante às células de Downey II, também chamadas de linfócitos amebóides, que são linfócitos circundantes aos eritrócitos, com citoplasma abundante, ocasionalmente com grânulos citoplasmáticos.

Os linfócitos plasmocitóides (Figura 2), descritos na primeira categoria, foram relatados por diversos estudos (Chong et al., 2020; Foldes et al., 2020; Jamal et al., 2020; Kaur et al., 2021; Lee \& Teo, 2020; Lüke et al., 2020; Nazarullah et al., 2020; Pozdnyakova et al., 2020; Sadigh et al., 2020; Schapkaitz, Jager \& Levy, 2020; Weinberg, Behdad \& Ji, 2020) revelando presença frequente no sangue periférico dos pacientes.

Figura 2. Linfócitos reativos presentes em pacientes com COVID-19, com características plasmocitóides.

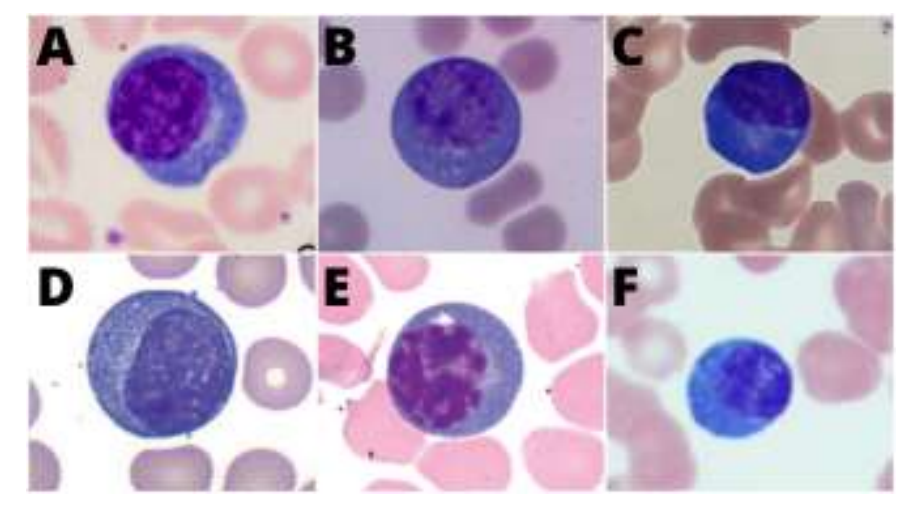

Fonte: compilação do autor ${ }^{1}$.

Os linfócitos reativos semelhantes às células de Downey (Figura 3), que mimetizam morfologicamente as células observadas nos pacientes infectados pelo vírus Epstein-Barr ou infecção por citomegalovírus (Gérard, Henry \& Thomas, 2020), foram observados com menos frequência (Berber et al., 2020; Chong et al., 2020; Gérard, Henry \& Thomas, 2020; Jamal et al., 2020; Lee \& Teo, 2020; Lüke et al, 2020; Nazarullah et al., 2020; Schapkaitz, Jager \& Levy, 2020).

${ }^{1}$ Fontes: A: Kaur et al., 2021. B: Schapkaitz, Jager \& Levy, 2020. C: Foldes et al., 2020. D: Pozdnyakova et al., 2020. E: Nazarullah et al., 2020. F: Jamal et al., 2020. 
Figura 3. Linfócitos reativos presentes em pacientes com COVID-19 com características semelhantes às células de Downey, ou linfócitos amebóides.

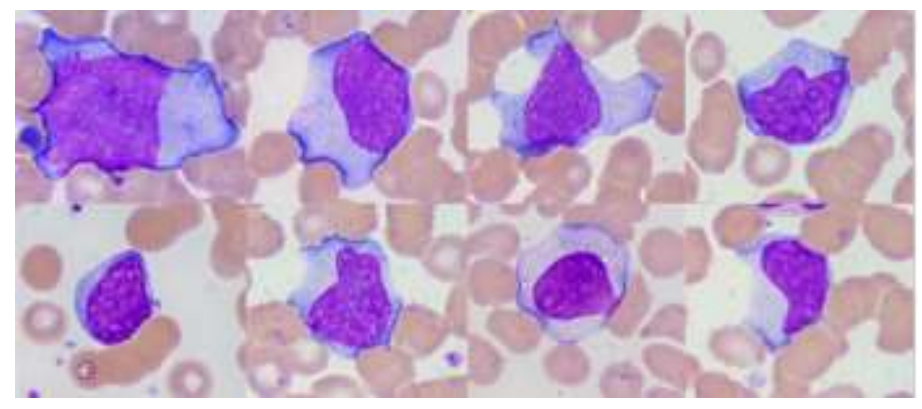

Fonte: Gérard, Henry \& Thomas (2020).

Outras morfologias citadas com menos frequência incluem a presença ocasional de grânulos citoplasmáticos (Gérard, Henry \& Thomas, 2020; Jamal et al., 2020; Kaur et al., 2021; Nazarullah et al., 2020; Schapkaitz, Jager \& Levy, 2020; Singh et al., 2020; Zhang H. et al., 2020) e linfócitos reativos com grandes inclusões citoplasmáticas, semelhantes às células de Mott (Foldes et al., 2020; Lee \& Teo, 2020; Lüke et al., 2020).

Singh et al. (2020), relatou várias anomalias. Foram vistos grandes linfócitos granulares, com núcleo recortado, cromatina condensada, nucléolos proeminentes e formação de cápsula citoplasmática. Células em apoptose também estavam presentes. Gérard, Henry e Thomas (2020) relataram a presença de linfócitos grandes (15-30 $\mu \mathrm{m})$, com basofilia, às vezes concentrada nas margens do citoplasma e às vezes generalizada. Células com nucléolos também foram observadas. Lüke et al. (2020) citou a presença de linfócitos altamente lobulados e alongados e referiu-se aos linfócitos reativos como “linfócitos aberrantes", visto que a sua morfologia se difere de outras infecções virais, como dengue e mononucleose infecciosa.

Os linfócitos reativos são um achado comum em esfregaços de sangue periférico de pacientes com COVID-19, mesmo no cenário de linfopenia. Os linfócitos plasmocitóides foram os achados mais comuns, o que indica sua relação com a infecção por SARS-CoV-2, visto que são incomuns em outras infecções virais causadoras de pneumonia, como Influenza A, SRAG-1 e gripe suína (Jamal et al., 2020).

Um estudo realizado por citometria de fluxo multicolor revelou que o número de linfócitos totais, células T, células B e células Natural Killer (NK) foram significativamente menores em pacientes com COVID-19 do que em comparação com indivíduos saudáveis, isso sugere uma relação entre a alteração do subconjunto de linfócitos com a COVID-19. As células NK desempenham papel importante contra infecções virais, pois causam a morte das células infectadas (Chan et al., 2020), impedindo a replicação viral.

A versão 7 do Protocolo de Diagnóstico e Tratamento para Pneumonia pelo Novo Coronavírus cita a linfopenia como um indicador clínico de alerta precoce para casos graves e críticos (National Health Commission \& National Administration of Traditional Chinese Medicine, 2020).

Tan L. et al. (2020), observaram em seu estudo que no decorrer de casos leves para casos graves, houve uma diminuição gradual da porcentagem de linfócitos circulantes. Quando a doença começou a melhorar, a porcentagem de linfócitos também começou a aumentar para níveis normais ou quase normais. Os pacientes que tiveram níveis persistentes de linfopenia, geralmente tinham um mau prognóstico. Blomme et al. (2020), também relatou o aumento dos linfócitos nos pacientes recuperados.

Nível baixo de linfócitos reflete em uma resposta imune prejudicada, isso faz com que os pacientes com COVID-19 sejam mais suscetíveis a infecção bacteriana associada e piora do quadro (Blomme et al., 2020; Sun S. et al., 2020). 
Em suma, os estudos relatados seguem a mesma linha de achados laboratoriais, o que confirma que a identificação da linfopenia e da morfologia dos linfócitos pode ser um achado importante frente a identificação e progressão da doença COVID-19.

\subsubsection{Monócitos}

Os monócitos são um subconjunto de leucócitos oriundos de progenitores mielóides, sofrem diferenciação na medula óssea e caem na circulação sanguínea. São células com tamanho entre 12 e $15 \mu \mathrm{m}$, com núcleo grande, citoplasma abundante e de coloração azul-acinzentada, com cromatina delicada. É comum encontrar vacúolos citoplasmáticos nos monócitos (Falcão \& Calado, 2001). Estas células são capazes de diferenciar-se em macrófagos e células dendríticas nos tecidos e desempenham papel importante na proteção do corpo contra infecções virais (Meidaninikjeh et al., 2021).

Os monócitos são células mononucleadas especializadas em respostas inflamatórias, fagocitose, apresentação de antígenos e liberação de citocinas (Nikitina et al., 2018). Estão presentes no sangue periférico e constituem cerca de 5 a $9 \%$ do total de leucócitos (Jafarzadeh et al., 2020). São divididos em três subgrupos principais, considerando a expressão dos marcadores CD14 e CD16. Os monócitos clássicos (CD14++ e CD16) que são os mais convencionais, os monócitos intermediários (CD14++ e CD16+) e os não clássicos (CD14+e CD16 ${ }^{++}$(Meidaninikjeh et al., 2021). Os monócitos não clássicos são "patrulheiros” que percorrem vasos sanguíneos para eliminar patógenos e células mortas. Quando em contato com agentes infecciosos, produzem citocinas e quimiocinas inflamatórias (Matic et al., 2020). No entanto, além do seu papel benéfico, podem representar atividade potencialmente patológica (Meidaninikjeh et al., 2021). Isso ocorre porque o SARS-CoV-2 pode desencadear uma desregulação imunológica, que resulta em ativação de monócitos e liberação descontrolada de citocinas (Nazarullah et al., 2020). Acredita-se que a desregulação de monócitos circulantes leva à expansão de monócitos inflamatórios e isso gera a ativação de citocinas e quimiocinas que aumentam a inflamação (Jafarzadeh et al., 2020). Ou seja, a ativação excessiva dos monócitos juntamente com a tempestade de citocinas desencadeia lesão pulmonar e consequente desenvolvimento da SRAG (Zhang H. et al., 2020).

Zhang H. et al. (2020), identificaram uma mudança distinta na morfologia e função dos monócitos, que foi associado à gravidade da doença e probabilidade de internação em unidade de terapia intensiva (UTI). Por citometria de fluxo foi possível identificar um aumento relativo de monócitos intermediários e não clássicos. Esses monócitos são células inflamatórias normalmente não vistas em pessoas saudáveis e que possuem grande capacidade de secretar citocinas próinflamatórias, o que os relaciona com a tempestade de citocinas. Ou seja, por citometria de fluxo, a simples avaliação do parâmetro Forward Scatter (FSC), que faz referência ao tamanho relativo da célula, pode indicar rapidamente os pacientes com aumento da secreção de citocinas e revelar o risco de internação em UTI além de complicações da doença.

Quanto à microscopia de rotina, foi relatado a presença de diferenças morfológicas e funcionais, principalmente, nos pacientes que requerem admissão em UTI (figura 4). Foram identificados monócitos maiores que o normal, com vacúolos atípicos, normalmente não vistos no sangue periférico de pacientes saudáveis (Kaur et al., 2021; Lüke et al., 2020; Pozdnyakova et al., 2020; Singh et al., 2020; Zhang H. et al., 2020). Já os pacientes recuperados ou com doença leve apresentam maior proporção de monócitos normais. Esse achado mostra-se específico para a COVID-19, visto que não foi relatado padrão similar em alguma outra doença viral, como Influenza ou HIV (Zhang H. et al., 2020). 
Figura 4. A: monócito de paciente saudável; B-H: monócitos displásicos, maiores que o normal com presença de grandes vacúolos citoplasmáticos, presentes em pacientes com COVID-19.

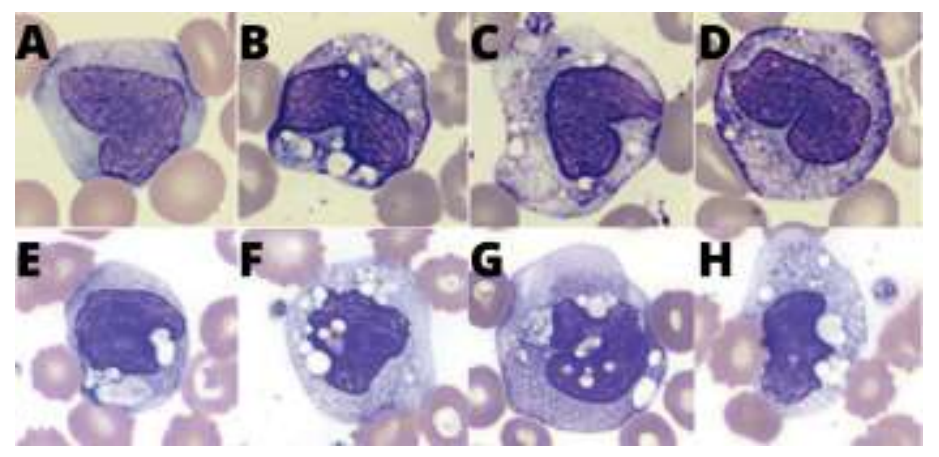

Fonte: compilação do autor ${ }^{2}$

A contagem de monócitos no sangue periférico varia de acordo com o estágio da doença e sofrem divergências nos diferentes estudos. Sun S. et al. (2020), relataram que a contagem de monócitos em pacientes internados na UTI foi menor do que em pacientes com doença leve. Matic et al. (2020) e Zhang D. et al. (2020), informaram que a contagem de monócitos estava dentro do intervalo de referência, porém, eram células maiores e estavam relacionadas a um fenótipo inflamatório. Já Pirsalehi et al. (2021), mostraram que houve monocitose nos pacientes em estado grave, no entanto, não foi uma descoberta estatisticamente significativa.

\subsubsection{Neutrófilos}

Os neutrófilos são as células imunes mais abundantes no sangue periférico, constituindo até $70 \%$ do total de leucócitos (Cavalcante-Silva et al., 2021). Fazem parte do sistema imunológico inato e são os primeiros a serem recrutados durante as infecções (Borges et al., 2020). Com sua morfologia característica são facilmente identificados e desempenham papel importante nas infecções bacterianas e fúngicas (Cavalcante-Silva et al., 2021). Em sua forma madura, os neutrófilos apresentam núcleo multilobulado (3 a 5 lóbulos) interligados por um filamento de cromatina. A cromatina é escura e densa e o citoplasma abundante, de coloração rosada e com presença de grânulos (Falcão \& Calado, 2001).

Sua principal função é a eliminação de patógenos e detritos celulares por meio da fagocitose, mas participam de vários outros processos, como por exemplo, a formação de armadilhas extracelulares de neutrófilos (neutrophil extracellular traps - NETs) que consistem em estruturas semelhantes a teias tridimensionais de ácidos nucléicos envoltos em histonas capaz de reter e degradar patógenos (Borges et al., 2020).

Durante o curso da COVID-19 os neutrófilos são ativados de forma persistente e isso desencadeia uma superprodução de NETs que acumulam-se nos tecidos e podem gerar oclusão vascular e microtrombose, não apenas no tecido pulmonar mas também no fígado e rins (Cavalcante-Silva et al., 2021). Portanto, a desregulação de NETs pode estar relacionada com a gravidade da doença e da lesão pulmonar (Borges et al., 2020).

Um dos parâmetros mais relatados é a presença de neutrofilia (Ahnach et al., 2020; Asghar M. S. et al., 2020; Chan et al., 2020; Kaur et al., 2021; Li et al., 2020; Liu, Zhang \& He, 2020; Lu \& Wang, 2020; Matic et al., 2020; Naoum et al., 2020; Nazarullah et al., 2020; Pozdnyakova et al., 2020; Sadigh et al., 2020; Sun D-W. et al., 2020; Sun S. et al.,2020; Zhang H. et al., 2020; Zingaropoli et al., 2021; Zini et al., 2020) que é caracterizada pelo aumento de neutrófilos circulantes no sangue periférico. Essa condição foi correlacionada com casos da doença grave, do qual o paciente necessitava de internação em UTI

\footnotetext{
2 Fontes: A-D: Zhang D. et al., 2020. E-H: Pozdnyakova et al., 2020.
} 
(Li et al., 2020), ou seja, à medida que o quadro do paciente piorava, a neutrofilia se intensificava (Liu, Zhang \& He, 2020; Pozdnyakova et al., 2020).

Quanto à morfologia, foram identificadas várias células displásicas (figura 5). Os neutrófilos exibiam presença de granulações tóxicas (Berber et al., 2020; Kaur et al., 2021; Nazarullah et al., 2020; Pozdnyakova et al., 2020; Salib \& TeruyaFeldsteins, 2020; Singh et al., 2020; Zhang H. et al., 2020; Zini et al., 2020), vacúolos citoplasmáticos (Kaur et al., 2021; Pozdnyakova et al., 2020; Singh et al., 2020; Zhang H. et al., 2020; Zini et al., 2020), núcleo hipersegmentado (Salib \& Teruya-Feldsteins, 2020) e múltiplas formas anormais, como núcleo em anel, em forma de C (Kaur et al., 2021; Singh et al., 2020; Zini et al., 2020).

Figura 5. A-C: neutrófilos de pacientes com COVID-19 com granulações tóxicas; D-F: neutrófilos com núcleos em formas anormais. Seta amarela: vacúolos citoplasmáticos e granulação tóxica; seta vermelha: núcleo em forma de anel.

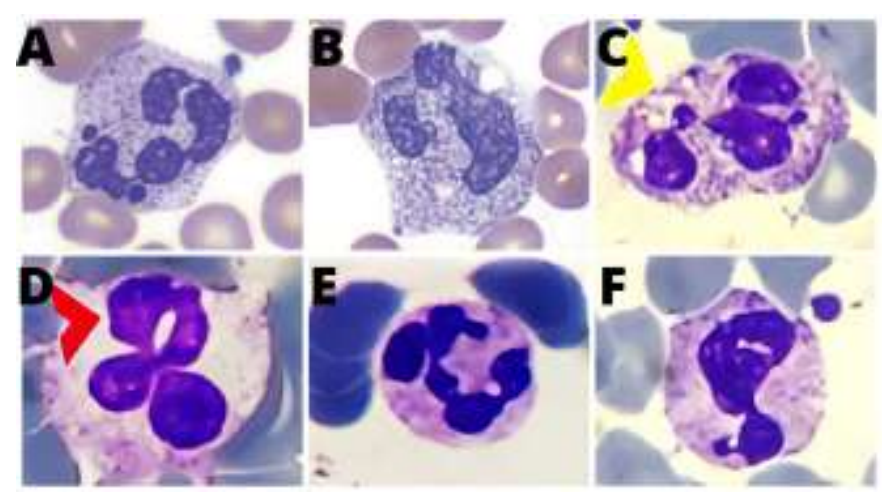

Fonte: compilação do autor ${ }^{3}$.

Outro achado relatado foi à presença de neutrófilos hipogranulares ou com áreas agranulares concentradas na periferia da célula (Ahnach et al., 2020; Berber et al., 2020; Pozdnyakova et al., 2020; Salib \& Teruya-Feldsteins, 2020; Zini et al., 2020). Anurag, Jha \& Kumar (2020), relataram que durante a pneumonia causada pelo SARS-CoV-2, como mecanismo de defesa, os neutrófilos tornam-se citotóxicos por meio da degranulação e lise, e isso podem agravar a imunopatologia da COVID-19.

Outra característica foi a presença de neutrófilos em desintegração que aparecem como formas manchadas (Kaur et al., 2021; Sadigh et al., 2020) e desvio a esquerda granulocítico, com a presença de promielócitos, mielócitos e metamielócitos no sangue periférico (Kaur et al., 2021; Mitra et al., 2020; Nazarullah et al., 2020; Pozdnyakova et al., 2020; Sadigh et al., 2020; Schapkaitz, Jager \& Levy, 2020; Zini et al., 2020).

Neutrófilos que apresentam defeitos na maturação que resulta em hipolobulação são chamados de Pseudo-Perger-Huet e foram observados por Ahnach et al. (2020); Berber et al. (2020); Kaur et al. (2021); Nazarullah et al. (2020) e Zini et al. (2020) (figura 6). A anomalia Perger-Huet é uma condição hereditária, mas o tipo adquirido está associado a várias patologias, como a Síndrome Mielodisplásica e abuso de drogas, mas nenhuma dessas condições foi observada nos pacientes com COVID-19 (Berber et al., 2020).

\footnotetext{
3 Fontes: A-B: Pozdnyakova et al., 2020. C-F: Singh et al., 2020.
} 
Figura 6. Neutrófilos com núcleos característicos da anomalia Pseudo-Pelger-Huet adquirida em COVID-19.

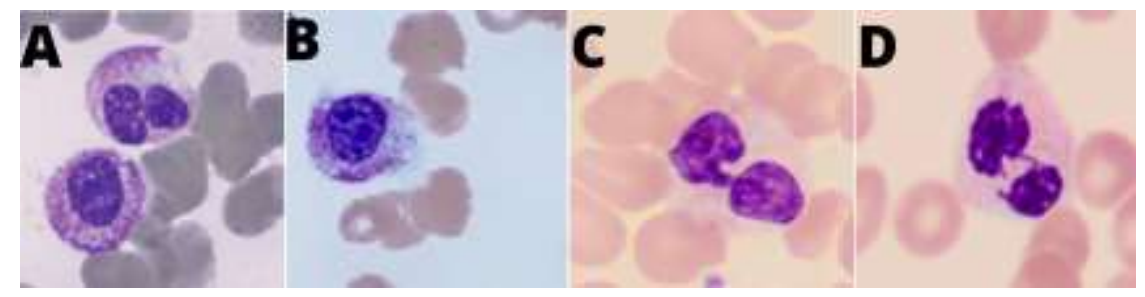

Fonte: compilação do autor ${ }^{4}$.

Quando analisados por citometria de fluxo exibiram tamanho significativamente maior, com presença de menos granulação em comparação com adultos saudáveis. Também estavam presentes neutrófilos maduros disfuncionais ou com fenótipo imaturo (Cavalcante-Silva et al., 2021; Middleton et al., 2020; Parackova et al., 2020).

Foi sugerido que a neutrofilia estaria associada a quadros de infecções bacterianas secundárias, devido à imunossupressão causada pelo vírus SARS-CoV-2, mas a maioria dos pacientes apresentava neutrofilia no estágio inicial da doença, dos quais não haviam sido detectadas infecções bacterianas (Li et al., 2020).

\subsubsection{Eosinófilos}

Os eosinófilos são um subconjunto de leucócitos granulócitos característicos por sua coloração alaranjada. Medem aproximadamente $8 \mu \mathrm{m}$ de diâmetro, possuem núcleo bilobado, citoplasma abundante rico em grânulos e compreendem cerca de 2 a 5\% do total de leucocitos (Rodrigo-Muñoz et al., 2021). Desenvolvem-se a partir de células progenitoras pluripotentes na medula óssea e quando maduros são liberados na corrente sanguínea onde permanecem por cerca de 1 a 2 dias antes de migrarem para os tecidos (Rosenberg \& Foster, 2021). Os eosinófilos são células importantes do sistema imunológico e aumentam em casos de infecções parasitárias e doenças alérgicas e tendem a diminuir em casos de doenças infecciosas agudas, como por exemplo, febre tifóide e sepse (Xie et al., 2020).

Quando estimulados, os eosinófilos migram para o local da inflamação, onde modulam as respostas do sistema imunológico e liberam diversas citocinas e quimiocinas. Eles também trabalham como células apresentadoras de antígenos não profissionais, processando e apresentando uma série de antígenos virais e parasitários (Rodrigo-Muñoz et al., 2021).

Os eosinófilos não são leucócitos fagocíticos, mas seus grânulos contêm uma grande quantidade de proteínas granulares catiônicas, citocinas e quimiocinas, fatores de crescimento, entre outros imunomoduladores, que contribuem para que os eosinófilos exerçam suas funções (Rodrigo-Muñoz et al., 2021).

A eosinopenia, que é caracterizada pela diminuição dos eosinófilos circulantes, foi a alteração mais relatada nos estudos (Ahnach et al., 2020; Berber et al., 2020; Chen et al., 2020; Djangang et al., 2020; Du et al., 2020; Liu F. et al., 2020; Naoum et al., 2020; Rosenberg \& Foster, 2021; Sun S. et al., 2020; Tan Y. et al., 2020; Tanni et al., 2020; Wu et al., 2020; Xie et al., 2020). Curiosamente, autores relataram a ausência absoluta de eosinófilos circulantes em pacientes confirmados com COVID-19 e internados na UTI (Tan Y. et al., 2020; Tanni et al., 2020; Wu et al., 2020). Pacientes com eosinopenia eram mais propensos a ter febre, dispnéia e, consequentemente, SRAG com evolução a óbito, quando comparados com pacientes com doença leve, que possuíam contagem de eosinófilos dentro do intervalo de referência. Portanto, a eosinopenia foi associada à gravidade da doença e indica maior chance de admissão na UTI (Xie et al., 2020).

A causa para essa eosinopenia é multifatorial, e inclui a diminuição da produção de leucócitos pela medula óssea, a migração dos leucócitos para o tecido pulmonar e o aumento do sequestro dentro da vasculatura, que diminui a sobrevida em circulação periférica (Sun D-W. et al., 2020; Tan Y. et al., 2020). A linfopenia também pode contribuir para a eosinopenia,

${ }^{4}$ Fontes: A-B: Zini et al., 2020. C-D: Kaur et al., 2021. 
visto que os linfócitos do subtipo Th2 são os responsáveis por produzir IL-5, que estimula e ativa a produção dos eosinófilos (Djangang et al., 2020). Porém, mais estudos são necessários para compreender o real mecanismo por trás deste processo.

Quando comparado com outras linhagens de leucócitos, os eosinófilos não apresentaram grandes mudanças em sua morfologia. Foram observados apenas múltiplos vacúolos citoplasmáticos (Ahnach et al., 2020; Beber et al., 2020; Pozdnyakova et al., 2020).

Como já mencionado, a eosinopenia persistente foi relacionada com a gravidade da doença, mas felizmente, estudos relatam que a contagem de eosinófilos circulantes retorna ao nível normal conforme os pacientes se recuperam da doença (Rosenberg \& Foster, 2021). A melhora contínua na contagem de eosinófilos foi sincronizada com a melhora das imagens radiológicas e dos sintomas (Chen et al., 2020; Liu F. et al., 2020; Rosenberg \& Foster, 2021). O que sugere que o aumento crescente dos eosinófilos é um sinal de recuperação da doença (Liu F. et al., 2020).

Em suma, a normalização na contagem dos eosinófilos sinaliza melhora do quadro clínico, enquanto a eosinopenia persistente é observada nos pacientes graves (Jesenak, Banovcin \& Diamant, 2020). Esse parâmetro pode ser levado em consideração quando há intenção de monitorar e evolução da doença, pois auxilia no prognóstico e na avaliação da eficácia do tratamento.

\subsubsection{Basófilos}

Os basófilos são o grupo de leucócitos granulócitos menos comuns, pois representam cerca de 0 a $1 \%$ do total de leucócitos (Siracusa et al., 2013). São característicos pela presença de grandes grânulos citoplasmáticos, que quando corados adquirem a cor púrpura escura. Morfologicamente, é uma célula com tamanho entre 10 e $15 \mu \mathrm{m}$, com citoplasma abundante, núcleo multilobulado com cromatina densa que, na maioria das vezes, está escondida sob os grânulos citoplasmáticos (Falcão \& Calado, 2001).

Esses leucócitos possuem tempo de vida por volta de 1 a 2 dias e também amadurecem na medula óssea sendo transferidos posteriormente para a corrente sanguínea onde exercem suas funções. Produzem diversos mediadores inflamatórios, principalmente histamina, e também possuem receptores IgE em sua superfície (Falcão \& Calado, 2001). A histamina fica armazenada nos seus grânulos citoplasmáticos e é liberada via estímulo, que pode ser mediado por citocinas, anticorpos ou pelos próprios antígenos (Siracusa et al., 2013).

Acredita-se que os basófilos contribuam com a patogênese de doenças alérgicas, rejeição de órgãos transplantados, doenças autoimunes e câncer, porém, essa relação ainda permanece mal compreendida (Siracusa et al., 2013).

A literatura é bastante inconsistente sobre a função dessa linhagem de leucócitos na patogênese da COVID-19. Berber et al. (2020); Chen et al. (2020); Mao et al. (2021) e Sun D-W. et al. (2020) relatam que, pacientes em casos graves tendem a ter menores contagens de basófilos. Chen et al. (2020) e Rodriguez et al. (2020) demonstraram que os basófilos são esgotados durante a fase aguda da doença grave, mas tendem a aumentar na fase de recuperação, sugerindo que estejam relacionados com o bom prognóstico. Já Anurag, Jha \& Kumar (2020), não correlacionam a contagem de basófilos com a COVID-19.

Há certa dificuldade em monitorar e relatar basopenia porque o valor de referência deste leucócito já é naturalmente baixo, portanto, levando em consideração os dados disponíveis até o presente momento, não podemos considerar a contagem de basófilos no sangue periférico como um marcador hematológico para o acompanhamento da COVID-19.

\section{Conclusão}

Anormalidade dos leucócitos circulantes em sangue periférico é comum em quadro de infecções virais, porém, o conjunto de anormalidades, numéricas e morfológicas, que foram observadas nos pacientes com COVID-19, são pouco frequentes em outras pneumonias causadas por infecções virais. Leucocitose, linfopenia, neutrofilia e eosinopenia, bem como, as alterações morfológicas dos linfócitos e neutrófilos, foram os achados mais consistentes no hemograma dos pacientes com 
COVID-19. A identificação dessas alterações não só fornece um significado auxiliar ao diagnóstico, mas também no direcionamento da evolução da infecção. Portanto, a contagem diferencial e avaliação morfológica de leucócitos é fundamental para evitar desfechos clínicos desfavoráveis.

Levando em consideração a instabilidade da pandemia e o surgimento de novas cepas variantes do SARS-CoV-2, recomenda-se para trabalhos futuros estudos e relatos de casos de infectados, com o objetivo de avaliar as possíveis mudanças na manifestação da doença e para manter as informações disponibilizadas sempre atualizadas.

\section{Referências}

Ahnach, M., Ousti, F., Nejjari, S., Houssaini, M. S., \& Dini, N. (2021). Peripheral Blood Smear Findings in COVID-19. Turkish Journal Of Hematology. 37(4), p. 301-302. http://dx.doi.org/10.4274/tjh.galenos.2020.2020.0262.

Amgalan, A., \& Othman, M. (2020). Hemostatic laboratory derangements in COVID-19 with a focus on platelet count. Platelets. 31(6), p. 740-745. http://dx.doi.org/10.1080/09537104.2020.1768523.

Anaurag, A., Jha, P. K., \& Kumar, A. (2020). Differential white blood cell count in the COVID-19: a cross-sectional study of 148 patients. Diabetes \& Metabolic Syndrome: Clinical Research \& Reviews. 14(6), p. 2099-2102. http://dx.doi.org/10.1016/j.dsx.2020.10.029.

Asghar, M., Hussain, N., Shoaib, H., Kim, M., \& Lynch, T. (2020). Hematological characteristics of patients in coronavirus 19 infection: a systematic review and meta-analysis. Journal Of Community Hospital Internal Medicine Perspectives. 10(6), p. 508-513, 29. http://dx.doi.org/10.1080/20009666.2020.1808360.

Asghar, M. S., Khan, N. A., Kazmi, S. J. H., Ahmed, A., Hassan, M., Jawed, R., Akram, M., Rasheed, U., Memon, G. M., Ahmed, M. U., Tahniyat, U., \& Tirmizi, S. B. (2020). Hematological parameters predicting severity and mortality in COVID-19 patients of Pakistan: a retrospective comparative analysis. Journal Of Community Hospital Internal Medicine Perspectives. 10(6), p. 514-520. http://dx.doi.org/10.1080/20009666.2020.1816276.

Berber, I., Casagar, O., Sarici, A., Berber, N. K., Aydogdu, I., Ulutas, O., Yildirim, A., Bag, H. G. G., \& Delen, L. A. (2020). Simple peripheral blood smear findings of COVID-19 patients provide information about the severity of the disease and the duration of hospital stay. Mediterranean Journal Of Hematology And Infectious Diseases. 13(1), p. 1-10. http://dx.doi.org/10.4084/mjhid.2021.009.

Blomme, S., Smets, L., Van Ranst, M., Boeckx, N., \& Van Laer, C. (2020). The influence of COVID-19 on routine hematological parameters of hospitalized patients. Acta Clinica Belgica. p. 1-6. http://dx.doi.org/10.1080/17843286.2020.1814649.

Borges, L., Pithon-Curi, T. C., Curi, R., \& Hatanaka, E. (2020). COVID-19 and Neutrophils: the relationship between hyperinflammation and neutrophil extracellular traps. Mediators Of Inflammation. p. 1-7. http://dx.doi.org/10.1155/2020/8829674.

Cavalcante-Silva, L. H. A., Carvalho, D. C. M., Lima, E. A., Galvão, J. G. F. M., Silva, J. S. F., Sales-Neto, J. M., \& Rodrigues-Mascarenhas, S. (2021). Neutrophils and COVID-19: the road so far. International Immunopharmacology. 90, p. 1-7. http://dx.doi.org/10.1016/j.intimp.2020.107233.

Celkan, T. T. (2020). Hemogram bize neler söyler? Türk Pediatri Arşivi. 55(2), p. 103-116. http://dx.doi.org/10.14744/turkpediatriars.2019.76301.

Chan, S. S. W., Christopher, D., Tan, G. B., Chong, V. C. L., Fan, B. E., Lin, C. Y., \& Ong, K. H. (2020). Peripheral lymphocyte subset alterations in COVID-19 patients. International Journal Of Laboratory Hematology. 42(5), p. 199-203. http://dx.doi.org/10.1111/ijlh.13276.

Chen, J., Pan, Y., Li, G., Xu, W., Zhang, L., Yuan, S., Xia, Y., Lu, P., \& Zhang, J. (2020). Distinguishing between COVID-19 and influenza during the early stages by measurement of peripheral blood parameters. Journal Of Medical Virology. 93(2), p. 1029-1037. http://dx.doi.org/10.1002/jmv.26384.

Choi, M., Aiello, E. A., Ennis, I. L., \& Villa-Abrille, M. C. (2020). El SRAA y el SARS-CoV-2: el acertijo a resolver. Hipertensión y Riesgo Vascular. 37(4), p. 169-175. http://dx.doi.org/10.1016/j.hipert.2020.05.005.

Chong, V. C. L., Lim, K. G. E., Fan, B. E., Chan, A. S. W., Ong, K. H., \& Kuperan P. (2020). Reactive lymphocytes in patients with COVID-19. British Journal Of Haematology. 189(5), p. 844-844. http://dx.doi.org/10.1111/bjh.16690.

Christensen, B., Favaloro, E. J., Lippi, G., \& Van Cott, E. M. (2020). Hematology Laboratory Abnormalities in Patients with Coronavirus Disease 2019 (COVID-19). Seminars In Thrombosis And Hemostasis. 46(07), p. 845-849. http://dx.doi.org/10.1055/s-0040-1715458.

Djangang, N. N., Peluso, L., Talamonti, M., Izzi, A., Gevenois, P. A., Garufi, A., Goffard, J-C., Henrard, S., Severgnini, P., Vincent, J-L., Creteur, J., \& Taccone, F. S. (2020). Eosinopenia in COVID-19 Patients: a retrospective analysis. Microorganisms. 8(12), p. 1-12. http://dx.doi.org/10.3390/microorganisms8121929.

Du, Y., Tu, L., Zhu, P., Mu, M., Wang, R., Yang, P., Wang, X., Hu, C., Ping, R., Hu, P., Li, T., Cao, F., Chang, C., Hu, Q., Jin, Y., \& Xu, G. (2020). Clinical Features of 85 Fatal Cases of COVID-19 from Wuhan. A Retrospective Observational Study. American Journal Of Respiratory And Critical Care Medicine. 201(11), p. 1372-1379. http://dx.doi.org/10.1164/rccm.202003-0543oc.

Estrela, C. (2018). Metodologia científica: ciência, ensino, pesquisa [recurso eletrônico]. 3 edição. Porto Alegre: Artes médicas. https://viewer.bibliotecaa.binpar.com/.

Falcão, R. P., \& Calado, R. T. (2001). Heterogeneidade das células do sangue: órgãos hematopoéticos e linfopoiéticos. In: Hematologia; Fundamentos e práticas. São Paulo: Atheneu, 2001. p. 1043-1053. https://repositorio.usp.br/item/001235332. 
Foldes, D., Hinton, R., Arami, S., \& Bain, B. (2020). Plasmacytoid lymphocytes in SARS-CoV -2 infection (Covid-19). American Journal Of Hematology. 95(7), p. 861-862, 28 abr. 2020. http://dx.doi.org/10.1002/ajh.25834.

Gérard, D., Henry, S., \& Thomas, B. (2020). SARS-CoV-2: a new aetiology for atypical lymphocytes. British Journal Of Haematology. 189(5), p. 845-845. http://dx.doi.org/10.1111/bjh.16730.

Helal, M. A., Shouman, S., Abdelwaly, A., Elmehrath, A. O., Essawy, M., Sayed, S. M., Saleh, A. H., \& El-Badri, N. (2020). Molecular basis of the potential interaction of SARS-CoV-2 spike protein to CD147 inOVID-19 associated-lymphopenia. Journal Of Biomolecular Structure And Dynamics. p. 1-11. http://dx.doi.org/10.1080/07391102.2020.1822208.

Jafarzadeh, A., Chauhan, P., Saha, B., Jafarzadeh, S. \& Nemati, M. (2020). Contribution of monocytes and macrophages to the local tissue inflammation and cytokine storm in COVID-19: lessons from sars and mers, and potential therapeutic interventions. Life Sciences. 257, p. 1-16. http://dx.doi.org/10.1016/j.1fs.2020.118102.

Jamal, S. M., Salib, C., Stock, A., \& Uriarte-Haparnas, N. I. (2020). Atypical lymphocyte morphology in SARS-CoV-2 infection. Pathology - Research And Practice. 216(9), p. 1-2. http://dx.doi.org/10.1016/j.prp.2020.153063.

Jesenak, M., Banovcin, P., \& Diamant, Z. (2020). COVID-19, chronic inflammatory respiratory diseases and eosinophils—Observations from reported clinical case series. Allergy. 75(7), p. 1819-1822. http://dx.doi.org/10.1111/all.14353.

Kaur, G., Sandeep, FNU., Olayinka, O., \& Gupta, G. (2021). Morphologic Changes in Circulating Blood Cells of COVID-19 Patients. Cureus. 13(2), p. 1-8, 18 fev. 2021. http://dx.doi.org/10.7759/cureus.13416.

Ke, Z., Oton, J., Qu, K., Cortese, M., Zila, V., McKeane, L., Nakane, T., Zivanox, J., Neufeldt, C. J., Cerikan, B., Lu, J. M., Peukes, J., Xiong, X., Kräusslich, H. G., Scheres, S. H. W., Bartenschlager, R., \& Briggs, J. A. G. (2020). Structures and distributions of SARS-CoV-2 spike proteins on intact virions. Nature. 588(7838), p. 498-502. http://dx.doi.org/10.1038/s41586-020-2665-2.

Khalil, O. A. K., \& Khalil, S. S. (2020). SARS-CoV-2: taxonomia, origem e constituição. Revista de Medicina. 99(5), p. 473-479. http://dx.doi.org/10.11606/issn.1679-9836.v99i5p473-479.

Khartabil, T. A., Russcher, H., Ven, A., \& Rijke, Y. B. (2020). A summary of the diagnostic and prognostic value of hemocytometry markers in COVID-19 patients. Critical Reviews In Clinical Laboratory Sciences. 57(6), p. 415-431. http://dx.doi.org/10.1080/10408363.2020.1774736.

Lee, C-T., Teo, W. Z. Y. (2020). Peripheral Blood Smear Demonstration of Lymphocyte Changes in Severe COVID-19. The American Journal Of Tropical Medicine And Hygiene. 103(4), p. 1350-1351. http://dx.doi.org/10.4269/ajtmh.20-0721.

Li, Q., Xie, Y., Cui, Z., Tang, S., Yuan, B., Huang, H., Hu, Y., Wang, Y., Zhou, M., \& Shi, C. (2020). Analysis of Peripheral Blood IL-6 and Leukocyte Characteristics in 364 COVID-19 Patients of Wuhan. Frontiers In Immunology. 11, p. 1-7. http://dx.doi.org/10.3389/fimmu.2020.559716.

Liao, D., Zhou, F., Lou, L., Xu, M., Wang, H., Xia, J., Gao, Y., Cai, L., Wang, Z., Yin, P., Wang, Y., Tang, L., Deng, J., Mei, H., \& Hu, Y. (2020). Haematological characteristics and risk factors in the classification and prognosis evaluation of COVID-19: a retrospective cohort study. The Lancet Haematology. 7(9), p. 671-678. http://dx.doi.org/10.1016/s2352-3026(20)30217-9.

Liu, F., Xu, A., Zhang, Y., Xuan, W., Yan, T., Pan, K., Yu, W., \& Zhang, J. (2020). Patients of COVID-19 may benefit from sustained Lopinavir-combined regimen and the increase of Eosinophil may predict the outcome of COVID-19 progression. International Journal Of Infectious Diseases. 95, p. 183-191. http://dx.doi.org/10.1016/j.ijid.2020.03.013.

Liu, X., Zhang, R., \& He, G. (2020). Hematological findings in coronavirus disease 2019: indications of progression of disease. Annals Of Hematology. 99 (7), p. 1421-1428. http://dx.doi.org/10.1007/s00277-020-04103-5.

Lu, G., \& Wang, J. (2020). Dynamic changes in routine blood parameters of a severe COVID-19 case. Clinica Chimica Acta, 508, p. 98-102. http://dx.doi.org/10.1016/j.cca.2020.04.034.

Lüke, F., Orsó, E., Kirsten, J., Poeck, H., Grube, M., Wolff, D., Burkhardt, R., Lunz, D., Lubnow, M., Schmidt, B., Hitzenbichler, F., Hanses, F., Salzberger, B., Evert, M., Herr, W., Brochhausen, C., Pukrop, T., Reichle, A., Heudobler, D. (2020). Coronavirus disease 2019 induces multi-lineage, morphologic changes in peripheral blood cells. Ejhaem, 1(1), p. 376-383. http://dx.doi.org/10.1002/jha2.44.

Mao, J., Dai, R., Du, R-C., Zhu, Y., Shui, L-P., \& Luo, X-H. (2021). Hematologic changes predict clinical outcome in recovered patients with COVID-19. Annals Of Hematology. 100(3), p. 675-689. http://dx.doi.org/10.1007/s00277-021-04426-x

Matic, S., Popovic, S., Djurdjevic, P., todorovic, D., Djordjevic, N., Mijailovic, Z., Sazdanovic, P., Milovanovic, D., Zecevic, D. R., Petrovic, M., Sazdanovic, M., Zornic, N., Vukiceic, V., Petrovic, I., Matic, S., Vukicevik, M. K., \& Baskic, D. (2020). SARS-CoV-2 infection induces mixed M1/M2 phenotype in circulating monocytes and alterations in both dendritic cell and monocyte subsets. Plos One. 15(12), p. 1-17. http://dx.doi.org/10.1371/journal.pone.0241097.

Meidaninikjeh, S., Sabouni, N., Marzouni, H. Z., Bengar, S., Khalili, A., \& Jafari, R. (2021). Monocytes and macrophages in COVID-19: friends and foes. Life Sciences. 269, p. 1-10. http://dx.doi.org/10.1016/j.lfs.2020.119010.

Middleton, E. A., He, X-Y., Denorme, F., Campbell, R. A., Ng, D., Salvatore, S. P., Mostyka, M., Baxter-Stoltzfus, A., Borczuk, A. C., Loda, M., Cody, M. J., Manne, B. K., Portier, I., Harris, E. S., Petrey, A. C., Beswick, E. J., Caulin, A. F., Iovino, A., Abegglen, L. M., ..., Yost, C. C. (2020). Neutrophil extracellular traps contribute to immunothrombosis in COVID-19 acute respiratory distress syndrome. Blood. 136(10), p. 1169-1179. http://dx.doi.org/10.1182/blood.2020007008.

Mina, A., Van Besien, K., \& Platanias, L. C. (2020). Hematological manifestations of COVID-19. Leukemia \& Lymphoma. 61(12), p. 2790-2798. http://dx.doi.org/10.1080/10428194.2020.1788017.

Mitra, A., Dwyre, D. M., Schivo, M., Thompson III, G. R., Cohen, S. H., Ku, N., \& Graff, J. P. (2020). Leukoerythroblastic reaction in a patient with COVID -19 infection. American Journal Of Hematology. 95(8), p. 999-1000. http://dx.doi.org/10.1002/ajh.25793. 
Naoum, F. A., Ruiz, A. L. Z., Martin, F. H. O., Brito, T. H. G., Hassem, V., \& Oliveira, M. G. (2020). Diagnostic and prognostic utility of WBC counts and cell population data in patients with COVID-19. International Journal Of Laboratory Hematology. p. 1-5. http://dx.doi.org/10.1111/ijlh.13395.

National Health Commission \& National Administration of Traditional Chinese Medicine. Diagnosis and Treatment Protocol for Novel Coronavirus Pneumonia (Trial Version 7). Chinese Medical Journal. 133(9), p. 1087-1095, 2020. https://doi.org/10.1097/CM9.0000000000000819.

Nazarullah, A., Liang, C., Villareal, A., Higgins, R. A., \& Mais, D. D. (2020). Peripheral Blood Examination Findings in SARS-CoV-2 Infection. American Journal Of Clinical Pathology. 154(3), p. 319-329. http://dx.doi.org/10.1093/ajcp/aqaa108.

Nikitina, E., Larionova, I., Choinzonov, E., \& Kzhyshkowska, J. (2018). Monocytes and Macrophages as Viral Targets and Reservoirs. International Journal Of Molecular Sciences. 19(9), p. 1-25. http://dx.doi.org/10.3390/ijms19092821.

OPAS - Organização Pan-Americana da Saúde. OMS afirma que COVID-19 é agora caracterizada como pandemia. 2020. Disponível em: https://www.paho.org/pt/news/11-3-2020-who-characterizes-covid-19-pandemic. Acesso em: 13 abr. 2021.

Parackova, Z., Zentsova, I., Bloomfield, M., Vrabcova, P., Smetanova, J., Klocperk, A., Meseznikov, G., Mendez, L. F. C., Vymazal, T., \& Sediva, A. (2020). Disharmonic Inflammatory Signatures in COVID-19: augmented neutrophils but impaired monocytes and dendritic cells responsiveness. Cells. 9(10), p. 1-17. http://dx.doi.org/10.3390/cells9102206.

Pirsalehi, A., Salari, S., Baghestani, A., Sanadgol, G., Shirini, D., Baerz, M. M., Abdi, S., Akbari, M. E., \& Bashash, D. (2021). Differential alteration trend of white blood cells (WBCs) and monocytes count in severe and non-severe COVID-19 patients within a 7-day follow-up. Iranian Journal Of Microbiology. 13(1), p. 8-16. http://dx.doi.org/10.18502/ijm.v13i1.5486.

Pozdnyakova, O., Cornnell, N. T., Battinelli, E. M., Connors, J. M., Fell, G., \& Kim, A. S. (2020). Clinical Significance of CBC and WBC Morphology in the Diagnosis and Clinical Course of COVID-19 Infection. American Journal Of Clinical Pathology. 155(3), p. 364-375. http://dx.doi.org/10.1093/ajcp/aqaa231.

Rodrigo-Muñoz, J. M., Sastre, B., Cañas, J. A., Gil-Martínez, M., Redondo, N., \& del Pozo, V. (2021). Eosinophil Response Against Classical and Emerging Respiratory Viruses: covid-19. Journal Of Investigational Allergology And Clinical Immunology. 31(2), p. 94-107. http://dx.doi.org/10.18176/jiaci.0624.

Rodriguez, L., Pekkarinen, P. T., Lakshmikanth, T., Tan, Z., Consiglio, C. R., Pou, C., Chen, Y., Mugabo, C. H., Nguyen, N. A., Nowlan, K., Strandin, T., Levanov, L., Mikes, J., Wang, J., Kantele, A., Hepojoki, J., Vapalahti, O., Heinonen, J., Kekäläinen, E., \& Brodin, P. (2020). Systems-Level Immunomonitoring from Acute to Recovery Phase of Severe COVID-19. Cell Reports Medicine. 1(5), p. 1-12. http://dx.doi.org/10.1016/j.xcrm.2020.100078.

Ropa, J., Cooper, S., Capitano, M. L., Hof, W. V., \& Broxmeyer, H. E. (2020). Human Hematopoietic Stem, Progenitor, and Immune Cells Respond Ex Vivo to SARS-CoV-2 Spike Protein. Stem Cell Reviews And Reports. 17(1), p. 253-265. http://dx.doi.org/10.1007/s12015-020-10056-z.

Rosenberg, H. F., \& Foster, P. S. (2021). Eosinophils and COVID-19: diagnosis, prognosis, and vaccination strategies. Seminars In Immunopathology. p. 110. http://dx.doi.org/10.1007/s00281-021-00850-3.

Sadigh, S., Massoth, L. R., Christensen, B. B., Stefely, J. A., Keefe, J., \& Sohani, A. R. (2020). Peripheral blood morphologic findings in patients with COVID-19. International Journal Of Laboratory Hematology. 42(6), p. 248-25. http://dx.doi.org/10.1111/ijlh.13300.

Salib, C., \& Teruya-Feldstein, J. (2020). Hypersegmented granulocytes and COVID-19 infection. Blood. 135(24), p. 2196-2196. http://dx.doi.org/10.1182/blood.2020006483.

Schapkaitz, E., Jager, T., Levy, B. (2020). The characteristic peripheral blood morphological features of hospitalized patients infected with COVID-19. International Journal Of Laboratory Hematology. 43(3), p. 130-134. http://dx.doi.org/10.1111/ijlh.13417.

Shahri, M. K., Niazkar, H. R., \& Rad, F. (2021). COVID-19 and hematology findings based on the current evidences: a puzzle with many missing pieces. International Journal of Laboratory Hematology. 43(2), p. 160-168. http://dx.doi.org/10.1111/ijlh.13412.

Silva, P. H., Alves, H. B., Comar, S. R., Henneberg, R., Merlin, J. C., \& Stinghen, S. T. (2016). Hematologia laboratorial: teoria e procedimentos. Porto Alegre: Artmed, 2016. 434 p. Disponível em: https://viewer.bibliotecaa.binpar.com/. Acesso em: 07 jul. 2021.

Singh, A., Sood, N., Narang, V., \& Goyal, A. (2020). Morphology of COVID-19-affected cells in peripheral blood film. Bmj Case Reports. 13(5), p. 1-2. http://dx.doi.org/10.1136/bcr-2020-236117.

Siracusa, M. C., Kim, B. S., Spergel, J. M., \& Artis, D. (2013). Basophils and allergic inflammation. Journal Of Allergy And Clinical Immunology. 132(4), p. 789-801. http://dx.doi.org/10.1016/j.jaci.2013.07.046.

Sun, D-W., Zhang, D., Tian, R-H., Li, Y., Wang, Y-S., Cao, J., Tang, Y., Zhang, N., Zan, T., Gao, L., Huang, Y-Z., Cui, C-L., Wang, D-X., Zheng, Y., \& Lv, G-Y. (2020). The underlying changes and predicting role of peripheral blood inflammatory cells in severe COVID-19 patients: a sentinel?. Clinica Chimica Acta. 508, p. 122-129. http://dx.doi.org/10.1016/j.cca.2020.05.027.

Sun, S., Cai, X., Wang, H., He, G., Lin, Y., Lu, B., Chen, C., Pan, Y., \& Hu, X. (2020). Abnormalities of peripheral blood system in patients with COVID-19 in Wenzhou, China. Clinica Chimica Acta. 507, p. 174-180. http://dx.doi.org/10.1016/j.cca.2020.04.024.

Tan, L., Wang, Q., Zhang, D., Ding, J., Huang, Q., Tang, Y-Q., Wang, Q., \& Miao, H. (2020). Lymphopenia predicts disease severity of COVID-19: a descriptive and predictive study. Signal Transduction And Targeted Therapy. (33). http://dx.doi.org/10.1038/s41392-020-0148-4.

Tan, Y., Zhou, J., Zhou, Q., Hu, L., \& Long, Y. (2020). Role of eosinophils in the diagnosis and prognostic evaluation of COVID-19. Journal Of Medical Virology. 93(2), p. 1105-1110. http://dx.doi.org/10.1002/jmv.26506.

Tanni, F., Akker, E., Zaman, M. M., Figueroa, N., Tharian, B., \& Hupart, K. H. (2020). Eosinopenia and COVID-19. Journal Of Osteopathic Medicine. (8), p. 504-508. http://dx.doi.org/10.7556/jaoa.2020.091. 
Research, Society and Development, v. 10, n.11, e400101119838, 2021

(CC BY 4.0) | ISSN 2525-3409 | DOI: http://dx.doi.org/10.33448/rsd-v10i11.19838

Walls, A. C., Park, Y-J., Tortorici, M. A., Wall, A., McGuire, A. T., \& Veesler, D. (2020). Structure, Function, and Antigenicity of the SARS-CoV-2 Spike Glycoprotein. Cell. (2), p. 281-292. http://dx.doi.org/10.1016/j.cell.2020.02.058.

Wang, M-Y., Zhao, R., Gao, L-J., Gao, X-F., Wang, D-P., \& Cao, J-M. (2020). SARS-CoV-2: structure, biology, and structure-based therapeutics development. Frontiers In Cellular And Infection Microbiology. 10(1), p. 1-17. http://dx.doi.org/10.3389/fcimb.2020.587269.

Weinberg, S. E., Behdad, A., Ji, P. (2020). Atypical lymphocytes in peripheral blood of patients with COVID-19. British Journal Of Haematology. 190(1), p. 36-39. http://dx.doi.org/10.1111/bjh.16848.

Wu, Y., Huang, X., Sun, J., Xie, T., Lei, Y., Muhammad, J., Li, X., Zeng, X., Zhou, F., Qin, H., Shao, L., \& Zhang, Q. (2020). Clinical Characteristics and Immune Injury Mechanisms in 71 Patients with COVID-19. Msphere. 5(4), p. 1-11. http://dx.doi.org/10.1128/msphere.00362-20.

Xie, G., Ding, F., Han, L., Yin, D., Lu, H., \& Zhang, M. (2020). The role of peripheral blood eosinophil counts in COVID-19 patients. Allergy. 76(2), p. 471482. http://dx.doi.org/10.1111/all.14465.

Zhang, D., Guo, R., Lei, L., Liu, H., Wang, Y., Wang, Y., Qian, H., Dai, T., Zhang, T., Lai, Y., Wang, J., Liu, Z., Chen, T., He, A., O’Dwyer, M., \& Hu, J. (2020). Frontline Science: covid-19 infection induces readily detectable morphologic and inflammation related phenotypic changes in peripheral blood monocytes. Journal Of Leukocyte Biology. 109(1), p. 13-22. http://dx.doi.org/10.1002/jlb.4hi0720-470r.

Zhang, H., Cao, X., Kong, M., Mao, X., Huang, L., He, P., Pan, S., Li, J., \& Lu, Z. (2020). Clinical and hematological characteristics of 88 patients with COVID-19. International Journal Of Laboratory Hematology. 42 (6), p. 780-787. http://dx.doi.org/10.1111/ijlh.13291.

Zingaropoli, M. A., Nijhawan, P., Carraro, A., Pasculli, P., Zuccalà, P., Perri, V., Marocco, R., Kertusha, B., Siccardi, G., Del Borgo, C., Curtolo, A., Ajassa, C., Iannetta, M., Ciardi, M. R., Mastroianni, C. M., \& Lichtner, M. (2021). Increased sCD163 and sCD14 Plasmatic Levels and Depletion of Peripheral Blood Pro-Inflammatory Monocytes, Myeloid and Plasmacytoid Dendritic Cells in Patients With Severe COVID-19 Pneumonia. Frontiers In Immunology. 12, p. 112. http://dx.doi.org/10.3389/fimmu.2021.627548. 